\title{
Exact Eigen Value Assignment of Linear Scalar Systems with Single Delay Using Lambert W Function
}

\section{${ }^{1}$ C.Y. Chew and ${ }^{2}$ H.N. Huang}

${ }^{1}$ Department of Mathematical \& Actuarial Sciences, Universiti Tunku Abdul Rahman, Bandar Sungai Long, Cheras 43000, Kajang, Selangor, Malaysia ${ }^{2}$ Department of Applied Mathematics, Tunghai University, Taichung 40704, Taiwan

Correspondence Author: C.Y. Chew, Department of Mathematical \& Actuarial Sciences, Universiti Tunku Abdul Rahman, Bandar Sungai Long, Cheras 43000, Kajang, Selangor, Malaysia.

Received date: 11 April 2018, Accepted date: 25 July 2018, Online date: 8 August 2018

Copyright: (C) 2018 C.Y. Chew and H.N. Huang. This is an open-access article distributed under the terms of the Creative Commons Attribution License, which permits unrestricted use, distribution, and reproduction in any medium, provided the original author and source are cre dited.

\begin{abstract}
Eigen value assignment problem of a linear scalar system with single discrete delay is analytically and completely solved. The existence condition on areal or complex desired eigenvalue is established when the current and delay states are included in the feedback. Design of the feedback controller is then followed by calculating the corresponding real feedback gains such that the rightmost eigenvalue of the closed-loop system is exactly located at the desired value. The eigenvalue assignment for the input-delay system is also obtained as well. Numerical examples illustrate the procedure in assigning the desired eigenvalue.
\end{abstract}

Key words: Lambert W Time-delay Eigenvalue assignment.

\section{INTRODUCTION}

During recent decades, the stabilization and control of linear systems with delays are extensively studied, for example, the spectrum (eigenvalues) assignment for linear delay systems in 1978[1]. Recently, an approach for the solution of a linear time-delay system by using the Lambert W function was proposed in [2]. Hence the robust stability as well as related topics to design the feedback controller are well established [3][4], and reference therein. A good introduction to the Lambert $\mathrm{W}$ function is given in [5] and this function possesses many applications within these two decades.

Eigenvalue assignment for delay systems with a single delay via Lambert W function is first developed in [6] to assign the rightmost eigenvalue of the delay system to a predefined (desired) location in order to stabilize the system, but unfortunately only a real or real part of the rightmost eigenvalue can be assigned for the scalar case. Alternatively, the assignment of the complex eigenvalue to the largest one of a scalar single delay system with the output of a complex feedback gain which is not realistic [7]. Later an analytic eigenvalue assignment method is proposed for scalar and some special delay systems [8]. All these studies design the controller by feedback only the current state and no condition is drawn on the value of desire eigenvalue such that the feedback controller always exists. On the other hand, although a more general time-delay system can be analyzed by using matrix Lambert $\mathrm{W}$ function, but the approach of computing the rightmost eigenvalue that is not using the principle branch contradicts the main proposition of this method [9].

Avoiding using the matrix Lambert $\mathrm{W}$ function, only a scalar system with single delay is considered in this paper. It mainly focuses on deriving the existence condition of the feedback controller related to assigning the rightmost eigenvalue of the system to the desired value. The formula to compute feedback gains for the current and delay states is then obtained. Furthermore, an input-delay type system is also considered. Two examples are provided to demonstrate the associated idea. The result consolidates further studies on the eigenvalue assignment of linear systems with multiple delays via the Lambert W function approach.

\section{LAMBERT W FUNCTIONS}

The Lambert $\mathrm{W}$ function is defined as a complex multivalued function which has an infinite number of branches, $W_{k}(z)$, where $k=0, \pm 1, \pm 2, \ldots, \pm \infty$ (regard $W_{\infty}$ and $W_{-\infty}$ as fixed mappings), such that

$W_{k}(z) e^{W_{k}(z)}=z, \quad z \in \mathbb{C}$

For any $x \in \mathbb{R}$, when $-1 / e \leq z=x<0$ the principal branch $W_{0}(x)$ satisfies $W_{0}(x) \geq-1$ and the -1 branch $W_{-1}(x)$ satisfies $W_{-1}(x) \leq-1$. By partitioning the $z$-plane with horizontal boundaries $z=(2 k+1) \pi$ for $k \in \mathbb{Z}$, the range of the $\operatorname{branch} W_{k}(z)$ is images of the $z$ between the branch cuts $z=$ $(2 k+1) \pi$ and $z=(2 k-1) \pi$ in the $z$-plane. $W_{0}$ has a branch cut whose image linking to $W_{1}$ and $W_{-1}$, is defined as $B C=\{x+i 0 \mid x \in(-\infty,-1 / e)\}$, i.e., $W_{0}(B C)$ is the boundary between ranges of $W_{0}$ and $W_{1}$ and so as $W_{-1}(B C)$ for $W_{-1}$ and $W_{0}$. The range of different branches as well as its real counterpart of this function is shown in Fig. 1.

An important property of the Lambert $\mathrm{W}$ function is proved by [7][10]:

Lemma 1:

Let $z \in \mathbb{C}$. Then $\overline{W_{k}(z)}=W_{-k}(\bar{z})$ and $\max _{k=0, \pm 1, \pm 2, \ldots, \pm \infty} \operatorname{Re} W_{k}(z)=\operatorname{Re} W_{0}(z)$. 
The first part of this lemma is also referred as the Lambert $\mathrm{W}$ function has the conjugate symmetricity which means the image of this function is symmetric about the real axis. The second part claims that the rightmost eigenvalue must be in the $W_{0}\left(\operatorname{and} W_{-1}\right.$ when it is complex). Consider the image of a circle $z=$ $r e^{i \theta}, \theta \in(-\pi, \pi]$ by $W$ as shown in Fig. 2 for three different cases: $r<1 / e, r=1 / e$, and $r>1 / e$ [4]. These graphs confirm $W_{0}$ contains the rightmost eigenvalue. Thus, this rule allows us to assign the rightmost eigenvalue by only considering the principal branch.

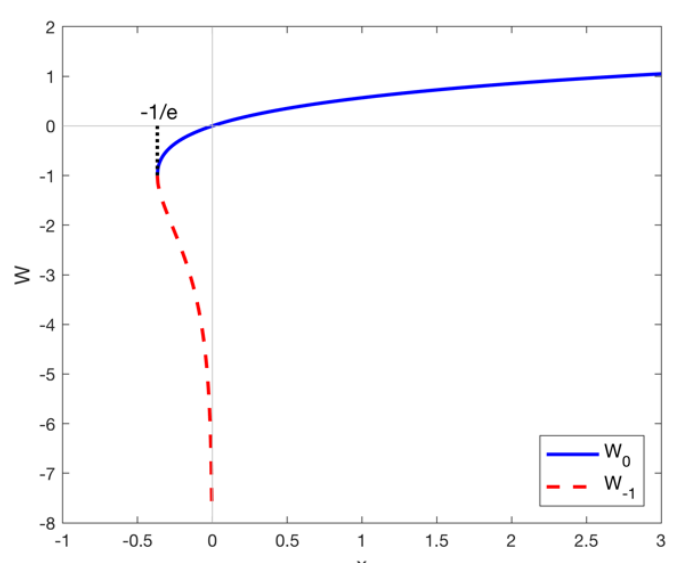

(a) $W_{0}(x), \stackrel{x}{W}-1(x), x \in \mathbb{R}$.

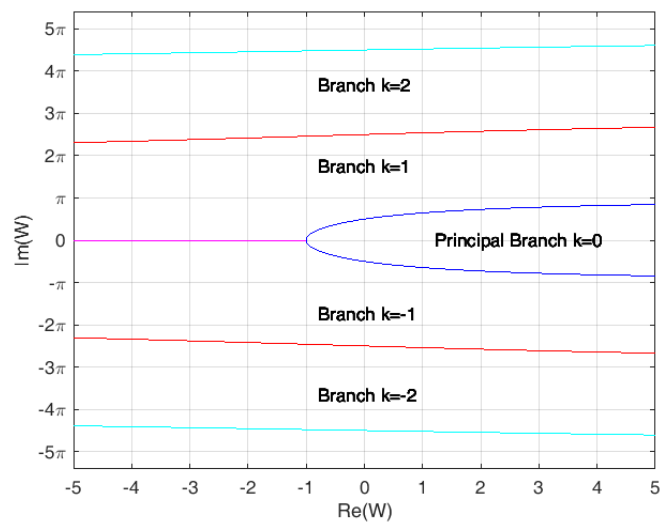

(b) Ranges of $W_{k}(z), k=0, \pm 1, \pm 2$.

Fig. 1: The range of Lambert $\mathrm{W}$ function.

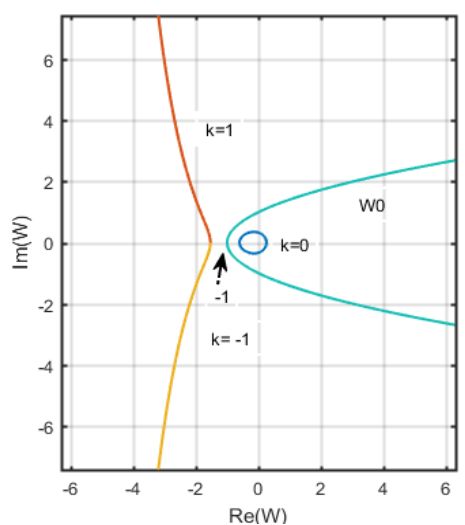

(a) $r<1 / e$

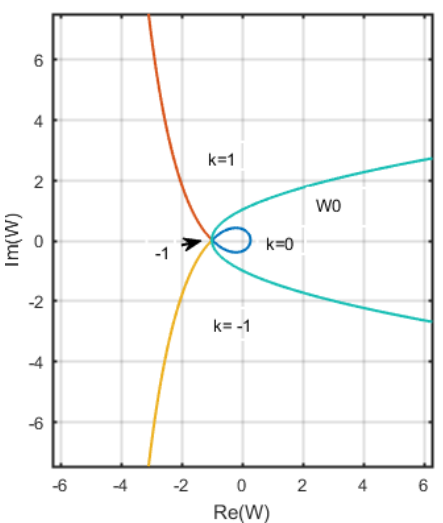

(b) $r=1 / e$

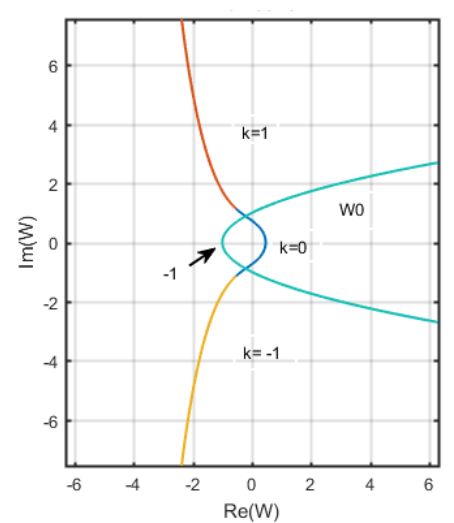

(c) $r>1 / e$

Fig. 2: Graphs of $W_{k}\left(r e^{i \theta}\right)$ with $k=0, \pm 1$ and $\theta \in(-\pi, \pi]$.

\section{MAIN RESULTS}

Consider a scalar delay system with an exogenous input from the environment:

$\dot{x}(t)=a x(t)+a_{1 d} x(t-h)+b u(t), h>0$,

$x(0)=x_{0}, x(\tau)=\phi(\tau),-h \leq \tau<0$,

where $a, a_{1 d}, b \neq 0, x_{0}, h \in \mathbb{R}$ and $\phi$ is the initial function to specify the state $x(\tau), \tau \in[-h, 0)$. Suppose a proportional control is proposed to stabilize the system with feedback of current and delay states:

$u(t)=k x(t)+k_{1 d} x(t-h)$

where two parameters $k, k_{1 d} \in \mathbb{R}$ areto be designed. The closed-loop system is then described by

$\dot{x}(t)=(a+b k) x(t)+\left(a_{1 d}+b k_{1 d}\right) x(t-h)$,

$$
\triangleq \alpha x(t)+\beta x(t-h)
$$

where $\alpha=a+b k$ and $\beta=a_{1 d}+b k_{1 d}$ are both real numbers.

The characteristic equation of the closed-loop system is given by [10]:

$f(s)=s-\alpha=\beta e^{-s h}$

or equivalently,

$s-(a+b k)=\left(a_{1 d}+b k_{1 d}\right) e^{-s h}$

whose roots (also known as eigenvalues of the system) are expressed by using Lambert W function

$s_{k}=\alpha+\frac{1}{h} W_{k}\left(\beta h e^{-\alpha h}\right), \quad k=0, \pm 1, \pm 2, \ldots, \pm \infty$

From Lemma 1, the system (3) is stable if and only if the real part of the rightmost eigenvalue, $s_{0}$, is negative.

The control design of a system with only input delay $(h>0)$, i.e. 
$\dot{x}(t)=a x(t)+b u(t-h), x(0)=x_{0}$

is a much more demanding challenge since the corresponding input-output operator is not compact. Applying the state feedback controller $u(t)=k x(t)$, then the associated closed-loop system becomes $\dot{x}(t)=a x(t)+b k x(t-h)$ which is also of the form (3) with $\alpha=a$ and $\beta=b k$. Therefore, we only need to focus on the system described by (3).

How to assign the rightmost eigenvalue $s_{0}$ of a closed-loop system to a desired location? Suppose the desired location is denoted by $S_{0, \text { des }} \in \mathbb{C}$ (with positive imaginary) but not a real number, we want to calculate real parameters $k$ and $k_{1 d}$ (i.e., adjust the values of $\alpha$ and $\beta$ ) such $s_{0}=S_{0, d e s}$.

One may approach the delay term in (4) with the first-order Padé approximation, i.e.:

$e^{-s h} \approx \frac{1-h s / 2}{1+h s / 2}$

As pointed out by [6] for the scalar system with single delay, this does assign a system's eigenvalue to $S_{0, d e s}$ but not for the rightmost eigenvalues. Thus, this approach is not suitable.

From (5) we have

$s_{0}=S_{0, d e s}=\alpha+\frac{1}{h} W_{0}\left(\beta h e^{-\alpha h}\right)$,

which means

$\alpha=S_{0, \text { des }}-\frac{1}{h} W_{0}\left(\beta h e^{-\alpha h}\right)$.

Define

$W_{0}^{\alpha} \triangleq\left\{S_{0, \text { des }}-\frac{1}{h} W_{0}(z) \mid z \in \mathbb{C}\right\}$.

whose graph is depicted in Fig.3. Hence for a given $S_{0, d e s}$, the parameter $\alpha$ must be picked from the set $W_{0}^{\alpha} \cap \mathbb{R}$. Once an $\alpha$ is selected, $\beta$ is then computed by

$\beta=\left(S_{0, \text { des }}-\alpha\right) e^{S_{0, d e s} h}$.

The approach has been proposed by [6] and [7]. But before carrying out this method, we must first answer the essential problem: what condition must $S_{0, d e s}$ satisfy in order to ensure the existence of the parameters $\alpha$ and $\beta$ so as the feedback gains $k_{1}$ and $k_{1 d}$ that the feedback controller exists.

Fig. 3: Graphs of the set $W_{0}^{\alpha}$.

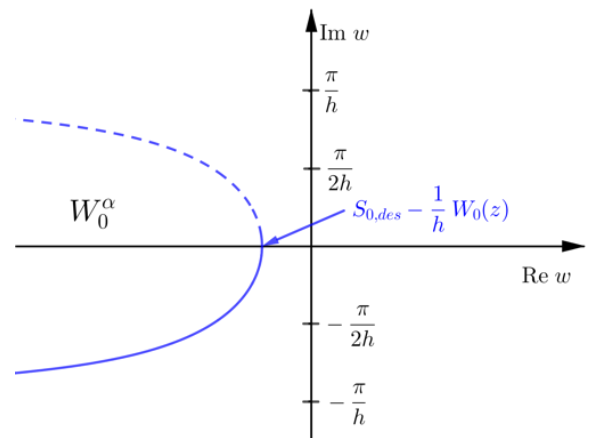

As discussed in [10], $\bar{S}_{0, \text { des }}$ must be the eigenvalue $s_{-1}$ of the system belongs to the range of $W_{-1}$. Let

$S_{0, d e s}=u+i v$

and substituting $s$ in (4) leads to

$u+i v-\alpha=\beta e^{-(u+i v) h}$

i.e.

$\left\{u-\alpha=\beta e^{-u h} \cos v h\right.$,

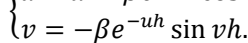

Combine to yield

$(u-\alpha) h=-v h \cot v h$

and hence,

$\left(S_{0, d e s}-\alpha\right) h=(u-\alpha) h+i v h \in W_{0}(B C)$

i.e., $\left(S_{0, \text { des }}-\alpha\right) h$ lies in the boundary between the ranges of $W_{0}$ and $W_{1}$. From (7) we have 
$\beta=-v e^{u h} \csc v h$

$\alpha=u+v \cot v h$

since $v \neq 0$, then the associated control feedback gains are given by

$\left\{\begin{aligned} k & =(u+v \cot v h-a) / b \\ k_{1 d} & =-\left(v e^{u h} \csc v h+a_{1 d}\right) / b .\end{aligned}\right.$

What happens when one of the states in (2) is not included in the feedback? Suppose the current state is not feedback $(k=0)$, the rightmost eigenvalue is assignable if

$\left(S_{0, \text { des }}-a\right) h \in W_{0}(B C)$

or equivalently, $a=u+v \cot v h$, and the delay state feedback gain is then given by

$k_{1 d}=\frac{\left(s_{0, d e s}-a\right) e^{S_{0, d e s} h}-a_{1 d}}{b}$.

Furthermore, suppose the delay state is not used $\left(k_{1 d}=0\right)$, we obtain

$S_{0, d e s}-a_{1 d} e^{-S_{0, d e s} h}=a+b k \in \mathbb{R}$

from (4), i.e., $S_{0, \text { des }}$ must satisfy the condition

$a_{1 d}+v e^{u h} \csc v h=0$.

The current state feedback gain is then described by

$k=\frac{S_{0, d e s}-a-a_{1 d} e^{-S_{0, d e s} h}}{b}$.

On the other hand, if $S_{0, \text { des }} \in \mathbb{R}$, then (5) becomes

$S_{0, \text { des }}=\alpha+\frac{1}{h} W_{k}\left(\beta h e^{-\alpha h}\right)$,

i.e.

$S_{0, \text { des }} \geq \alpha-\frac{1}{h}$, while $\beta h e^{-\alpha h} \geq-\frac{1}{e}$

due to the range of $W_{0}$ lies in the right-hand side of the vertical line $u=-1$. Unless the system is an input-delay system, we always and find an $\alpha$ (or $k$ ) such that

$\alpha \leq S_{0, \text { des }}+\frac{1}{h}$

$\beta=\left(S_{0, d e s}-\alpha\right) e^{S_{0, d e s} h}$

with corresponding

$k=\frac{\alpha-a}{b}, \quad k_{1 d}=\frac{\beta-a_{1 d}}{b}$

i.e,

$\left\{\begin{aligned} k & \leq \frac{S_{0, d e s}-a}{b}+\frac{1}{b h}, \\ k_{1 d} & =\frac{\left[S_{0, d e s}-(a+b k)\right] e^{S_{0, d e s}}}{b}-\frac{a_{1 d}}{b} .\end{aligned}\right.$

Suppose the current state is not present in the feedback $(k=0)$, the rightmost eigenvalue is assignable if $S_{0, \text { des }} \geq a-1 / h$, and the delay state feedback gain is still given by (9). Similarly, when the delay state is not used $\left(k_{1 d}=0\right)$, we have $S_{0, d e s}-a_{1 d} e^{-S_{0, d e s} h}=a+b k$ which is always achievable bythe feedback gain from (10).

The above derivation answers the existence question on $S_{0, \text { des }}$ such that the eigenvalue assignment can be performed. It encapsulates as follows:

Theorem 2:

Suppose the system (1) is not an input-delay system, the following statements hold:

(i) For a given $S_{0, \text { des }}=u+i v \in \mathbb{C}$, the rightmost eigenvalue of the system (3) can be assignable to any desired location $S_{0, \text { des }}$ via the controller (2) with both current- and dealy-state feedback gains defined by (8). Furthermore, if the current or delay state is not included in the feedback loop, the $S_{0, d e s}$ must satisfy the condition

$a=u+v \cot v h$

or

$a_{1 d}=-v e^{u h} \csc v h$

such that the associated gain is described by (9) or (10), respectively. 
(ii) For a given $S_{0, \text { des }} \in \mathbb{R}$, the rightmost eigenvalue of the system (3) can be assignable to any desired location $S_{0, \text { des }}$ via the controller (2) with feedback gains defined by (11). Furthermore, if the current or delay state is not included in the feedback loop, the $S_{0, \text { des }}$ must satisfy the condition

$S_{0, d e s} \geq a-\frac{1}{h}$

or no constraint such that the associated gain is still described by (9) or (10), respectively.

When the desired eigenvalue $S_{0, \text { des }} \in \mathbb{R}$, there are two free parameters $k$ and $k_{1 d}$ to be determined. Since only one equation (4) needs to hold, hence one more eigenvalue, say $S_{1, \text { des }}<S_{0, \text { des }} \in \mathbb{R}$, can be assigned. Under this circumstance it follows that

$S_{0, \text { des }}-\alpha=\beta e^{-S_{0, d e s} h}$,

$S_{1, \text { des }}-\alpha=\beta e^{-S_{1, d e s} h}$.

Suppose $S_{0, \text { des }} \neq S_{1, \text { des }}$, then one obtains

$\alpha=\frac{S_{1, \text { des }} e^{-S_{0, d e s} h}-S_{0, \text { des }} e^{-S_{1, d e s} h}}{e^{-S_{0, d e s}}-e^{-S_{1, d e s}}}$,

$\beta=\frac{S_{0, \text { des }}-S_{1, \text { des }}}{e^{-S_{0, \text { des }} h}-e^{-S_{1, d e s} h}}$.

Since $S_{0, \text { des }}$ must be assigned to the rightmost eigenvalue of the closed-loop system, i.e.,

$S_{0, d e s}=\alpha+\frac{1}{h} W_{0}\left(\beta h e^{-\alpha h}\right)$

and suppose that $S_{1, d e s}$ is located into the range of a certain branch, say the $k$-th branch of the Lambert $\mathrm{W}$ function:

$S_{1, d e s}=\alpha+\frac{1}{h} W_{k}\left(\beta h e^{-\alpha h}\right)$.

Let $z_{w}=\beta h e^{-\alpha h} \in \mathbb{R}$, then it is obvious that both $W_{0}\left(z_{w}\right)$ and $W_{k}\left(z_{w}\right)$ must be real, and hence $-1 / e<z_{w}<0$ and $k=-1$. Thus

$S_{0, d e s}=\alpha+\frac{1}{h} W_{0}\left(\beta h e^{-\alpha h}\right)$,

$S_{1, \text { des }}=\alpha+\frac{1}{h} W_{-1}\left(\beta h e^{-\alpha h}\right)$,

Where

$-\frac{1}{e}<\beta h e^{-\alpha h}<0$.

This sufficient condition turns out to be

$\alpha h>1+\log (-\beta h)$,

or equivalently,

$\frac{S_{1, d e s} h e^{-S_{0, d e s} h}-S_{0, d e s} h e^{-S_{1, d e s} h}}{e^{-S_{0, d e s}}-e^{-1_{1, d e s}}}>1+\log \frac{S_{1, d e s} h-S_{0, d e s} h}{e^{-S_{0, d e s}}-e^{-S_{1, d e s}}}$.

Once this condition is satisfied, the feedback controller exists, and the associated gains are described by

$\left\{\begin{aligned} k & =\frac{\left(S_{1, d e s}-a\right) e^{-S_{0, d e s}}-\left(S_{0, d e s}-a\right) e^{-S_{1, d e s}}}{\left(e^{-S_{0, d e s}}-e^{-S_{1, d e s}}\right) b}, \\ k_{1 d} & =\frac{S_{0, d e s^{-}} S_{1, d e s}}{\left(e^{\left.-S_{0, d e s^{h}}-e^{-S_{1, d e s}}\right) b}-\frac{a_{1 d}}{b} .\right.}\end{aligned}\right.$

Alternatively considering $S_{1, \text { des }}=S_{0, \text { des }}$, i.e., the closed-loop system will have two eigenvalues to be assigned into the same location, i.e., $s_{0}=S_{0, \text { des }}$, and $s_{-1}=S_{0, \text { des }}$. We take the limit $S_{1, \text { des }} \rightarrow S_{0, \text { des }}$ onthe above result which give us

$\alpha=S_{0, \text { des }}+\frac{1}{h}$,

$\beta=-\frac{1}{h} e^{S_{0, d e s} h}$,

And

$\left\{\begin{aligned} k & =\frac{S_{0, d e s}-a}{b}+\frac{1}{b h}, \\ k_{1 d} & =-\frac{1}{b h} e^{S_{0, d e s} h}-\frac{a_{1 d}}{b} .\end{aligned}\right.$

At the same time, we have 
$\beta h e^{-\alpha h}=-\frac{1}{e}$

which means the parameter $\alpha$ must be selected to compensate the distance from $S_{0, \text { des }}$ to $-1=W(-1 / e)$ such that the parameter $\beta$ move $x$ to $-1 / e$ where the $W_{0}$ and $W_{-1}$ branches contact each other (see Fig. 1(a)).It is obvious that (14) is the limit case of (11) with the equality holds.

When $S_{0, \text { des }} \in \mathbb{R}$, there is another possibility to design the controller by selecting

$k=\frac{S_{0, d e s}-a}{b}, k_{1 d}=-\frac{a_{1 d}}{b}$

i.e., the closed-loop system becomes

$\dot{x}(t)=S_{0, \text { des }} x(t)$

whose solution is given by

$x(t)=x_{0} e^{S_{0, d e s} t}$.

This approach can get rid of the delay state by using the feedback controller to compensate for the delay effect. The above discussion on the real eigenvalue assignment can be summarized as follows: Corollary 3.

Suppose the system (1) is not an input-delay system and $S_{0, \text { des }}, S_{1, \text { des }} \in \mathbb{R}$, the following statements hold:

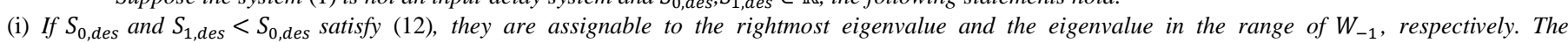
corresponding feedback gains are the described by (13).

(ii) If the feedback gains given by(14) is adopted, then the closed-loop system (3)has $S_{0, \text { des }}$ to be its rightmost eigenvalue with multiplicity 2.

(iii) If the feedback gain (15) is adopted, the closed-loop system has $S_{0, \text { des }}$ as its eigenvalue and this system becomes delay-free. An input-delay system (6) is assignable to any complex $S_{0, d e s}$ if

$\left(S_{0, \text { des }}-a\right) h \in W_{0}(B C)$,

i.e.

$\left(S_{0, \text { des }}-a\right) h e^{\left(S_{0, d e s}-a\right) h}=z$

for some real number $z<-1 / e$. Then the associated real feedback gain for the controller $u=k x(t)$ is determined by

$k=\frac{\left(S_{0, d e s}-a\right) e^{S_{0, d e s} h}}{b}$.

Or when $S_{0, \text { des }}$ is real, it must satisfy

$S_{0, \text { des }} \geq a-\frac{1}{h}$

and the feedback gain is still given by (16) which is the same as the result presented in [7]. Thus for the input-delay system, the assertion becomes

Corollary 4: obtained. For an input-delay system (6), if $S_{0, \text { des }}-\alpha$ belongs to the upper boundary on the range of $W_{0}$ or $[-1, \infty)$, a real feedback gain $k$ through (16) is

Example 1:

Consider the system (1) with the given data set $a=1, a_{1 d}=-1, b=1$, and $h=1$. Determine the values of $k$ and $k_{1 d}$ such that the rightmost closedloop eigenvalue $s_{0}$ is located at $S_{0, \text { des }}$ which is equal to $-0.092484+1.9973 \mathrm{i},-0.60502+1.7882 \mathrm{i}$, or -1 , respectively. By Theorem 2 and Corollary 3 , this system is eigenvalue-assignable to three desired eigenvalues with the feedback gains given by (8) for the first two and (15) for the third one. Table 1 shows the computational result for these parameters. And the corresponding variation of characteristic roots of the closed-loop system before and after the eigenvalue assignment are also shown in Table 2 by using the method proposed in [10]. For the third eigenvalue, the closed-loop system becomes a non-delay system which has only one eigenvalue -1 as expected.

Table 1: State feedback gains of the controller with respect to three different eigenvalue assignments.

\begin{tabular}{|l|l|l|l|}
\hline$S_{0, \text { des }}$ & $-0.092484+1.99730 \mathrm{i}$ & $-0.60502+1.78820 \mathrm{i}$ & $-1.0+0 \mathrm{i}$ \\
\hline$k$ & -2 & -2 & -2 \\
\hline$k_{1 d}$ & -1 & 0 & 1 \\
\hline
\end{tabular}

Example 2:

Consider an input-delay system (6) with $a=-1, b=2$ and $h=1$. By Corollary 4 , when $S_{0, \text { des }}=-2=a-1 / h$ located at the boundary of the admissible region, thus $k=-1 / 2 e^{2} \approx-0.0677$; and when $S_{0, \text { des }}=-2+i$ which is not in $W_{0}(B C)$ thus, this eigenvalue is not assignable.

Table 2: The variation of characteristic roots before and after eigenvalue assignment.

\begin{tabular}{|l|l|l|}
\hline$a=1, \quad a_{1 d}=-1$ & $k=-2, \quad k_{1 d}=-1$ & $k=-2, \quad k_{1 d}=0$ \\
\hline$s_{3,-3}=-3.02630 \pm 20.2238 \mathrm{i}$ & $s_{3,-4}=-2.32231 \pm 20.3555 \mathrm{i}$ & $s_{3,-4}=-3.01658 \pm 20.3214 \mathrm{i}$ \\
\hline$s_{2,-2}=-2.66407 \pm 13.8791 \mathrm{i}$ & $s_{2,-3}=-1.95315 \pm 14.0695 \mathrm{i}$ & $s_{2,-3}=-2.64736 \pm 14.0202 \mathrm{i}$ \\
\hline$s_{1,-1}=-2.08880 \pm 7.46150 \mathrm{i}$ & $s_{1,-2}=-1.36300 \pm 7.80750 \mathrm{i}$ & $s_{1,-2}=-2.05280 \pm 7.71840 \mathrm{i}$ \\
\hline$s_{0}=0$ & $s_{0,-1}=-0.092484 \pm 1.99730 \mathrm{i}$ & $s_{0,-1}=-0.60502 \pm 1.78820 \mathrm{i}$ \\
\hline
\end{tabular}




\section{CONCLUSIONS}

The sufficient condition on the solving the eigenvalue assignment of a linear scalar systems with single delay is analytically developed. The feedback controller is designed by using both the current and delay states. Furthermore, similar sufficient condition for input-delay systems is also depicted. The gains to associate feedback states are computed accordingly such that the closed-loop system behaves the same as expected. Examples are presented for an illustrate purpose. This result provides more flexibility in real-world applications than previous studies.

\section{ACKNOWLEDGEMENT}

This work was partially supported by the Ministry of Science \& Technology, Taiwan, under the grant No. MOST 106-2115-M-029-004.The second named author gratefully acknowledge the hospitality of the Mathematical \& Actuarial Sciences, Sungai Long Campus, Universiti Tunku Abdul Rahman, Malaysia.

\section{REFERENCES}

[1] Olbrot, A.W., 1978. Stabilization, Detectability, and Spectrum Assignment for Linear Autonomous Systems with General Time Delays, IEEE Trans. Autom. Control, 23(5): 887-890.

[2] Asl, F.M. and A.G. Ulsoy, 2003. Analysis of a system of linear delay differential equations, ASME J. Dyn. Sys., Meas., Control, 125(2): 215-223.

[3] Yi, S., P.W. Nelson and A.G. Ulsoy, 2010. Time-Delay Systems: Analysis and Control Using the Lambert W Function, World Scientific.

[4] Shinozaki, H. and T. Mori, 2006. Robust Stability Analysis of linear time-delay Systems by Lambert W Function, Automatica, 42: 1791-1799.

[5] Corless, R.M., G.H. Gonnet, D.E.G. Hare, D.J. Jeffrey and D.E. Knuth, 1996. On the Lambert W function, Adv. Comput. Math., 5: 329-359.

[6] Yi, S., P.W. Nelson and A.G. Ulsoy, 2010. Eigenvalue Assignment via the Lambert W Function for Control of Time-Delay Systems, J. Vib. Control., 16: 961-982.

[7] Shinozaki, H., 2007. Lambert W Function Approach to Stability and Stabilization Problems for Linear Time-Delay System, Thesis, Kyoto Institute of Technology.

[8] Nemati, M., F. Hashemzadeh, M. Baradarannia and A.R. Chiasi, 2017. An analytical Eigenvalue Assignment of Linear Time-Delay Systems using Lambert W function, in Proc. 5th International Conference on Control, Instrumentation, and Automation (ICCIA), pp: 222-227.

[9] Cepeda-Gomez, R. and W. Michels, 2015. Some special cases in the stability analysis of multi-dimensional time-delay system using the matrix Lambert W function, Automatica, 53: 339-345.

[10] Huang, H.N., 2017. Characteristic Roots of a Linear Scalar System with Multiple Delays, in Proc. 13th IEEE International Conference on Control \& Automation (ICCA), pp: 341-346. 